\title{
Heavy Ion Fragmentation Reactions at Energies of 35-140 MeV in a Combined Transport and Statistical Approach
}

\author{
Tatiana Mikhailova $^{1, \star}$, Batchuluun Erdemchimeg ${ }^{1,2}$, Anatoliy Artukh $^{1}$, Yuriy Sereda ${ }^{1}$, \\ Massimo Di Toro ${ }^{3}$, and Hermann H. Wolter ${ }^{4}$ \\ ${ }^{1}$ Joint Institute for Nuclear Research, Dubna, 141980 Russia \\ ${ }^{2}$ Mongolian National University, NRC, P.O.B 46A/305, Ulaanbaatar, Mongolia \\ ${ }^{3}$ Lab. Naz. del Sud, INFN, I-95123 Catania, Italy \\ ${ }^{4}$ Faculty of Physics, University of Munich, Garching, 85748 Germany
}

\begin{abstract}
Fragment formation in heavy ion collisions at low to intermediate energy is described by a combined application of transport theory of the Boltzmann type and of a statistical program for the decay of the fragments at the late stage. The transport equations are solved by simulations using the test particle method as a finite element representation of the phase space distribution. The description of experimental data is reasonable overall, but the fragment velocity distributions point to the presence of other mechanisms and the role of fluctuations.
\end{abstract}

\section{Introduction}

The phase diagram of matter interacting by the strong force as a function of density and temperature has a rich variety of phases and phase transitions. Heavy ion collision at various energies can be used to explore this. Here we investigate the liquid-gas phase transition in nuclear matter, which is closely connected to the process of fragmentation in heavy ion reactions, where in the final expansion phase a decomposition into intermediate mass fragments and a nucleon gas may take place. This phase transition is of theoretical interest, but fragment formation also has practical applications in the production of, possibly exotic, isotopes, and generally for accelerator-driven technologies (ADS). However, heavy ion collisions are non-equilibrium processes and have to be interpreted by non-equlibrium approaches. Hydrodynamical or statistical methods can be used in parts of the process, but a universal description requires transport theory, which is a microscopic description using effective interactions. The solution of transport theories is a challenging computational problem in many part of physics. We discuss fragment production in a two-stage approach consisting of the formation of primary hot fragments, and the de-excitation to cold fragments, yielding isotope distributions and their energy or velocity spectra. Thus we attempt to understand the mechanism of the fragment production in a microscopic and systematic way in reactions of various systems and incident energies.

\footnotetext{
^e-mail: tmikh@jinr.ru
} 


\section{Theoretical and Computational Considerations}

The BNV, also often called Boltzmann-Uehling-Uhlenbeck (BUU), transport approach describes the time evolution of the one-body phase space distribution function $f(\vec{r}, \vec{p}, t)$ under the influence of a self-consistent mean field $U([f])$ and a Boltzmann two-body collision term, which includes the effect of Pauli blocking [1].

$$
\begin{aligned}
& \frac{\partial f_{1}}{\partial t}+\frac{\overrightarrow{p_{1}}}{m} \nabla_{\vec{r}} f_{1}-\nabla_{\vec{r}} U \nabla_{\overrightarrow{p_{1}}} f_{1}= \\
& \left(\frac{2 \pi}{m}\right)^{3} \int d \vec{p}_{2} d \vec{p}_{3} d \vec{p}_{4}\left|\vec{v}_{1}-\vec{v}_{2}\right| \sigma_{N N}\left(\Omega_{12}\right) \delta\left(\vec{p}_{1}+\vec{p}_{2}-\vec{p}_{3}-\vec{p}_{3}\right)\left(f_{3} f_{4} \bar{f}_{1} \bar{f}_{2}-f_{1} f_{2} \bar{f}_{3} \bar{f}_{4}\right) .
\end{aligned}
$$

Here $f_{i}=f\left(\vec{r}, \vec{p}_{i}, t\right), \bar{f}_{i}=\left(1-f_{i}\right), v_{i}=p_{i} / m$ are velocities, $\sigma_{N N}(\Omega)$ is the in-medium NN cross-section, and $\mathrm{m}$ is the nucleon mass. The solution of this non-linear integro-differential equation is achieved by simulations using the test particles (TP) method, where the distribution function is represented in terms of finite elements as $f(\vec{r}, \vec{p} ; t)=1 / N_{T P} \cdot \sum_{n=1}^{A N_{\mathrm{TP}}} g\left(\vec{r}-\vec{r}_{n}(t)\right) \tilde{g}\left(\vec{p}-\vec{p}_{n}(t)\right)$, where $N_{\mathrm{TP}}$ is the number of TP per nucleon (of the order of 50-100; 50 in this work), $\vec{r}_{n}$ and $\vec{p}_{n}$ are the time-dependent coordinates and momenta of the test particles, and $g$ and $\tilde{g}$ are the shape functions in coordinate and momentum space (e.g. $\delta$-functions or Gaussians), respectively. Upon inserting this ansatz into the 1.h.s. of eq. (1) Newtonian equations of motion for the test particles follow $m \frac{\mathrm{d}^{2} \vec{r}_{n}}{\mathrm{~d} t^{2}}=-\vec{\nabla}_{r_{n}} U$, which are solved by the leapfrog method ( here with a time step of $0.5 \mathrm{fm} / \mathrm{c}$ ). The collision term is simulated stochastically, by performing test particle collisions with a probability depending on the cross section and checking the Pauli principle for the final state. The potential $U([f])$ and the cross-section are either derived from an energy density functional, or are parametrized in order to test them relative to the data (here a Skyrme functional and parametrized cross sections [2]).

Fragments are identified in terms of a coalescence criterion in coordinate and momentum space. The time evolution of the reaction is followed until the freeze-out time when the different fragments are sufficiently isolated so that nuclear forces between them become negligible. Then the intrinsic energy of each fragment, and thus its excitation energy, is calculated consistently using the same energy functional that determines the potential $U$ in the transport equation. These 'primary' fragments are hot fragments, i.e. they are excited by several $\mathrm{MeV}$ per nucleon depending on the impact parameter. They are propagated to infinity analytically by the Coulomb interaction between projectile- and target-like fragments. Then the de-excitation is calculated using a statistical decay code, in our case the Statistical Multifragmentation Model (SMM) [3], where we consider evaporation, Fermi-breakup, and multifragmentation. Thus we obtain the isotope distributions and velocity spectra of the final cold fragments, which are compared to experimental data.

\section{Results}

Here we intend to show to which extent we are able to describe isotope distributions and their velocity spectra using the microscopic transport theory sketched above. We consider reactions, which cover a range of incident energies from 35 to $140 \mathrm{AMeV}$ per nucleon and a variety of projectiles always on the same target, namely ${ }^{181} \mathrm{Ta}$. In particular, these are the projectiles ${ }^{18} \mathrm{O}$ at $35 \mathrm{AMeV} \mathrm{[4,2];}{ }^{40} \mathrm{Ar}$ at 57 $\mathrm{AMeV}[5,6] ;{ }^{40,48} \mathrm{Ca}$ at $140 \mathrm{AMeV}[7,8]$, and ${ }^{64} \mathrm{Ni}$ at $140 \mathrm{AMeV}$ [7]. Here the first reference refers to the data, the second to our previous analyses. The calculation for the last reaction is presented here.

In Fig. 1 we show the isotope distributions for the element with charge number $Z_{\text {proj }}-2$ (in panel (f) also for the light element $\mathrm{Mg}$ from the ${ }^{64} \mathrm{Ni}$ reaction) for the hot fragments (BNV, circles) and the cold fragments (BNV+SMM, squares) in comparison to the corresponding data (triangles). For 

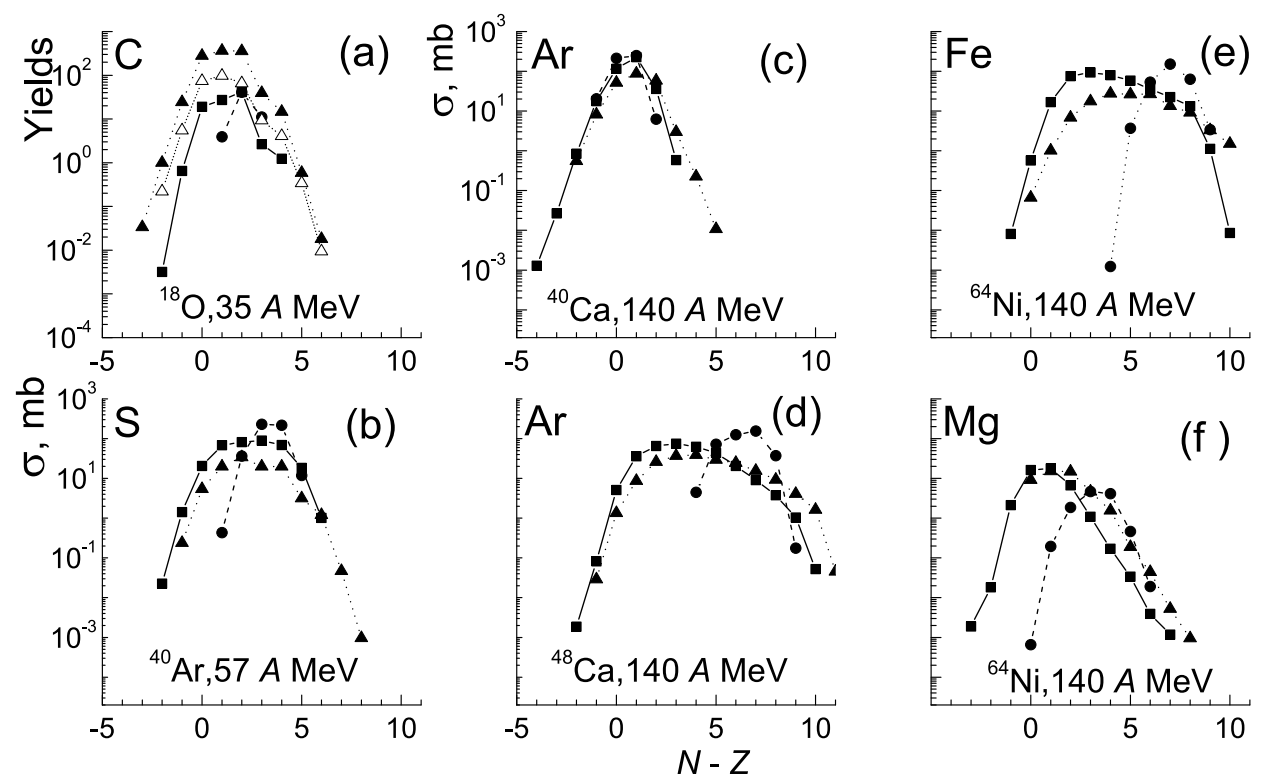

Figure 1. Isotope distributions for different reactions on the target ${ }^{181} \mathrm{Ta}$. The projectile and the incident energy are identified in each panel at the bottom. For each reaction we give the isotope distribution for one particular element identified in the upper left corner of each panel. The lines are: BNV (solid circle, dashed line), BNV+SMM (solid square, solid line), experiment (solid triangle, dotted line). In panel (a) also the experimental dissipative component is given (see text, open triangle, dotted line)

${ }^{18} \mathrm{O}$ we show an extra dissipative curve explained below. The hot fragments have a rather narrow distribution, which is centered around the $N-Z$ of the projectile. In the de-excitation of the fragments predominantly neutrons are evaporated, and the distribution of the cold fragments is shifted towards the line of stability. The agreement with experiment is reasonable considering that this is a microscopic calculation without fitted parameters.

In Fig. 2 we give, for the same reactions and the same elements as in Fig. 1, the velocity spectra as a function of the reduced velocity $v_{\text {red }}=v / v_{\text {proj }}$, summed over the isotopes of the given element. The experimental distributions peak at $v_{\text {red }}=1$, while the calculations generally peak below 1 , as particularly evident for the ${ }^{18} \mathrm{O}$ reaction in panel (a). We assume that the experiment contains a component with almost no loss of velocity, as could be expected in a direct abrasion-like process or in multi-nucleon transfer, which a semi-classical transport theory cannot describe. To separate this contribution from the experiment we subtract a Gaussian which is fitted to the distribution for $v_{\text {red }}>1$. The calculations are seen to be in better agreement with this dissipative curve. The direct component decreases with higher energy as seen in the other panels, where we have not subtracted it, but where it is still somewhat apparent. The width of the BNV distributions, i.e. the velocity fluctuations, is considerably narrower than the data. By including the de-excitation it widens and is now in better agreement especially on the the low velocity side, though still usually too narrow. This is particularly evident for the $\mathrm{C}$ spectrum for the ${ }^{18} \mathrm{O}$ reaction in panel (a), which has a large low velocity tail. Thus the fluctuations in the transport calculations are not large enough. It is known that the Boltzmann theory does not contain realistic physical fluctuations, which should be described by an explicit fluctuation term in the Boltzmann-Langevin theory. 

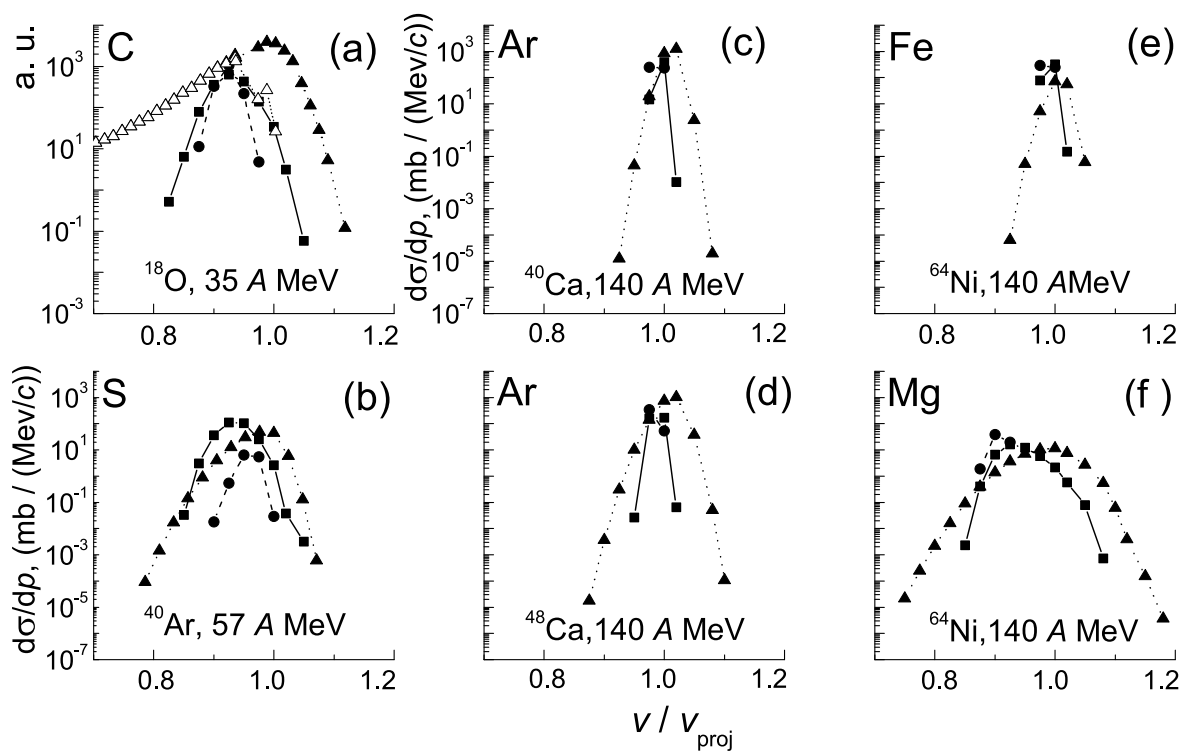

Figure 2. Velocity spectra of the same reactions for the same elements as in Fig. 1 as a function of the reduced velocity. The signatures are also the same as in Fig. 1.

\section{Conclusion}

We present a systematic study of heavy ion fragmentation reactions using microscopic transport theory, combined with a statistical code for the de-excitation of the primary hot fragments. Generally this can describe the isotope distributions fairly well. The velocity spectra show finer details of the reaction mechanism, but are too narrow, probably due to too small fluctuations. However, the approach appears to be useful to estimate fragment production in heavy ion collisions.

\section{Acknowledgements}

The authors thank A. Botvina for providing us the computer code SMM and for usefull advice. This work was supported by the Heisenberg-Landau Program. TM is supported by the Russian Foundation for Basic Research under Grant N 17-01-00661-a, and HHW by the Universe Cluster of Excellence of the DFG Germany.

\section{References}

[1] G. F. Bertsch, and S. Das Gupta, Phys. Rep. 160, 189 (1988)

[2] T. I. Mikhailova et al., Phys. Part. Nucl. Lett. 12, 409 (2015)

[3] J. P. Bondorf, A. Botvina, et al. Phys. Rep. 257, 133 (1995); A. Ergun et al. Phys. Rev. C 92, 014610 (2015)

[4] A. G. Artukh et al., Nucl.Phys. A 701, 96c (2002)

[5] X. H. Zhang et al., Phys. Rev. C 85, 024621 (2012)

[6] T. I. Mikhailova et al., Bull. of RAS: Physics, 78, 1131 (2014)

[7] M. Mocko et al., Phys. Rev. C 78, 024612 (2008)

[8] T. I. Mikhailova et al., Phys. Atomic Nuclei, 79, 604 (2016) 